УДК 519.21

\title{
Intermediate Systems and Higher-Order Differential Constraints
}

\author{
Oleg V. Kaptsov* \\ Institute of Computational Modelling SD RAS \\ Academgorodok, 50/44, Krasnoyarsk, 660036 \\ Russia
}

Received 10.03.2018, received in revised form 20.04.2018, accepted 06.06.2018

A method for constructing solutions of nonlinear partial differential equations with two independent variables is proposed. The method is based on the search for so-called intermediate systems, each solution of which satisfies the initial equation. The main attention is paid to a second order nonlinear wave equation. We give examples of intermediate systems and corresponding solutions.

Keywords: differential constraints, defining equations, invariant manifolds.

DOI: 10.17516/1997-1397-2018-11-5-550-560.

\section{Introduction}

One of the first methods of integrating nonlinear partial differential equations, proposed by Monge and Ampère, can be formulated as follows [1,2]. Suppose we are given an equation of the second order

$$
\Phi\left(t, x, u, u_{t}, u_{x}, u_{t t}, u_{t x}, u_{x x}\right)=0 .
$$

It is necessary to find an equation of the first order

$$
V\left(t, x, u, u_{t}, u_{x}\right)+c=0,
$$

depending on an arbitrary constant $c \in \mathbb{R}$ such that any non-singular solution satisfies (1). The equation (2) is called the first or intermediate integral of the second order equation (1). Further development of this method was obtained in the works of Darboux and his followers. The key point in the Darboux method is that it is necessary to find an equation of order $n$ that depends on a constant and is in involution with (1). The details of the Monge-Ampére and Darboux methods are well described in [3]. Modern aspects of these methods and some generalizations are presented in $[4,5]$. It should be noted that the equations (1), to which the Monge-Ampère and Darboux methods are applicable, are exceptional. For example, among the equations of the form

$$
u_{t x}=f(u)
$$

only the Liouville equation is integrated by the Darboux method. On the other hand, the Goursat problem of describing all equations of the form

$$
u_{t x}=F\left(t, x, u, u_{t}, u_{x}\right)
$$

integrable by the Darboux method remains open [6].

In this paper, we propose an approach to the integration of equations of the type (1), based on the finding of such systems

$$
u_{t}=G\left(x, u, u_{1}, \ldots, u_{m}\right), \quad u_{i}=\frac{\partial^{i} u}{\partial x^{i}},
$$

*kaptsov@icm.krasn.ru

(c) Siberian Federal University. All rights reserved 


$$
H\left(x, u, u_{1}, \ldots, u_{n}\right)=0,
$$

that each solution of this system satisfies the equation (1) as well. We suppose the manifold (5) is invariant under the equation (4) [7] and say that the system (4), (5) is intermediate.

The article has the following structure. In Section 2 we construct intermediate systems for equations of the type (3) using their higher symmetries. The higher symmetries [8] are also called generalized or Lie-Backlund symmetries $[9,10]$. It is shown that if the equation $(3)$ admits an operator of higher symmetry

$$
\eta \frac{\partial}{\partial u}+\sum_{n=1}^{\infty} D_{x}^{n}(\eta) \frac{\partial}{\partial u_{n}}
$$

where $D_{x}$ is the operator of total derivative with respect to $x$, then the system

$$
u_{t}+\eta=0, \quad D_{x}(\eta)+f=0
$$

is intermediate for the equation (3). To solve the system (6), we use the Runge-Kutta methods for the approximate solutions of ordinary differential equations. Some solutions of the sine-Gordon equation are given. In the third section, intermediate systems for the equation (3) are found without the use of symmetries. It turns out that the equation

$$
u_{t}=e^{u}\left(u_{2}-u_{1}^{2}\right)
$$

has a simultaneous solution with the sinh-Gordon equation, and the third order evolution equation

$$
u_{t}=e^{u}\left(u_{3}-2 u_{1} u_{2}\right)
$$

has a simultaneous solution with the Tzitzeica equation.

The fourth section is devoted to the construction of invariant manifolds for diffusion equations

$$
u_{t}=\left(u^{k} u_{x}\right)_{x} .
$$

For special exponents $k$ we found differential constraints depending on the third, fourth and fifth derivatives with respect to $x$. Examples of exact solutions of the equation (7) obtained by integrating differential constraints are given.

\section{Intermediate systems generated by symmetries}

Among the nonlinear equations of the form

$$
u_{t x}=f(u),
$$

as is known [11], only equations with right hand sides $f=a_{1} \exp (u)+a_{2} \exp (-u), f=a_{1} \sin (u)$, $f=a_{1} \exp (u)+a_{2} \exp (-2 u)\left(a_{1}, a_{2} \in \mathbb{R}\right)$ have higher symmetries.

We recall that the operator

$$
X=\eta \frac{\partial}{\partial u}+\sum_{n=1}^{\infty} D_{x}^{n}(\eta) \frac{\partial}{\partial u_{n}}
$$

is a higher symmetry of the equation (8) if the equality

$$
D_{t} D_{x} \eta=\eta \frac{\partial f}{\partial u}
$$

is satisfied according to the equation (8) and its differential consequences. Here and thereafter, $D_{t}, D_{x}$ will mean the operators of total derivatives with respect to $t$ and $x$ respectively [9]. 
To each operator $X$ one can associate the differential equation

$$
\eta=0
$$

This leads to the system (8), (9) for the function $u$. In this section we consider examples of such systems. We begin with the Tzitzeica equation

$$
u_{t x}-\exp (u)-\exp (-2 u)=0 .
$$

It has a denumerable set of higher symmetries [11]. Let us investigate the compatibility of a system consisting of (10) and the equation

$$
u_{5}+5\left(u_{2} u_{3}-u_{1}^{2} u_{3}-u_{1} u_{2}^{2}\right)+u_{1}^{5}=0 .
$$

Here and thereafter, we will use the notation $u_{i}=\frac{\partial^{i} u}{\partial x^{i}}$. The left-hand side of (11) defines a fifth order higher symmetry of the Tzitzeica equation (10). From the system (10), (11) you can get more convenient one. Indeed, differentiating (11) with respect to $t$ and substituting the mixed derivatives, according to (11), we obtain a new equation

$$
u_{4}-\frac{u_{1} u_{3}\left(e^{3 u}+4\right)+3 u_{2}^{2}\left(-e^{3 u}+1\right)+2 u_{1}^{2} u_{2}\left(2 e^{3 u}-1\right)-u_{1}^{4}\left(e^{3 u}+1\right)}{e^{3 u}-2}=0 .
$$

Further, differentiating the last equation with respect to $t$ and substituting the mixed derivatives from (11), we obtain the evolution equation

$$
u_{t}+\frac{\left(2 e^{-2 u}-e^{u}\right)\left(u_{3}+2 u_{1} u_{2}\right)}{2 u_{1} u_{3}-\left(u_{2}-u_{1}^{2}\right)^{2}}=0 .
$$

By direct calculations one can verify that the equation (12) is an invariant manifold of the evolution equation (13).

We recall the notion of the invariant manifold [7]. Let us consider an evolution equation

$$
u_{t}+F\left(t, x, u, u_{1}, \ldots, u_{n}\right)=0
$$

and an ordinary differential equation

$$
h \equiv u_{m}+G\left(x, u, u_{1}, \ldots, u_{m-1}\right)=0 .
$$

The equations (14) and (15) form the manifolds in the jet space [8].

Definition 1. The manifold (15) is called invariant under the equation (14) if the relation

$$
D_{t} h=0
$$

is satisfied due to (14), (15) and their differential consequences with respect to the variable $x$. In this case, the system (14), (15) will be called passive (in the narrow sense).

Remark. The general definition of a passive system in the analytic case is given in [12].

It should also be noted that the Tzitzeica equation is a differential consequence of the system (10), (11). More precisely, the following formula holds

$$
D_{x}\left(e_{1}\right)+\frac{\left(2 e^{-2 u}-e^{u}\right)\left(u_{2}+u_{1}^{2}\right)^{2}}{\left(2 u_{1} u_{3}-\left(u_{2}-u_{1}^{2}\right)^{2}\right)^{2}} e_{2}=u_{t x}-\exp (u)-\exp (-2 u),
$$

where $e_{1}$ is the left-hand side of (13), $e_{2}$ is the left-hand side of (12). Hence, any solution of the system (12), (13) is a solution of the Tzitzeica equation. 
If we set four initial conditions

$$
\frac{\partial^{i} u}{\partial x^{i}}\left(t_{0}, x_{0}\right)=c_{i}, \quad i=0, \ldots, 3, \quad c_{i} \in \mathbb{R}
$$

then from [5] it follows that the system (12) and (13) will have a unique local solution. It is assumed that the initial data are not special, i.e. denominators in (12) and (13) are not equal to zero. We say that the solution of the system depends on four constants in the neighborhood of a nonsingular point.

As a second example, consider the sine-Gordon equation

$$
u_{t x}-\sin (u)=0
$$

supplemented by an ordinary differential equation of the fifth order

$$
u_{5}+\frac{5}{2}\left(u_{1}^{2} u_{3}+u_{1} u_{2}^{2}\right)+\frac{3}{8} 8 u_{1}^{5}=0
$$

generated by one of the higher symmetries. Repeating the arguments given above for the Tzitzeica equation, from the last two equations we obtain the passive system

$$
\begin{gathered}
e_{3} \equiv u_{4}+\left(12 u_{2} u_{1}^{2}+8 u_{3} u_{1} \operatorname{tg}(u)-4 u_{2}^{2}+3 u_{1}^{4} \operatorname{tg}(u)\right) / 8=0, \\
e_{4} \equiv u_{t}+4 \cos (u) \frac{2 u_{3}+u_{1}^{3}}{8 u_{3} u_{1}-4 u_{2}^{2}+3 u_{1}^{4}}=0 .
\end{gathered}
$$

The solution of this system also depends on four constants, and the sin-Gordon equation is a differential consequence of this system

$$
D_{x}\left(e_{4}\right)+\frac{8 \cos (u)\left(4 u_{2}^{2}+u_{1}^{4}\right)}{\left(8 u_{3} u_{1}-4 u_{2}^{2}+3 u_{1}^{4}\right)^{2}} e_{3}=u_{t x}-\sin (u) .
$$

Definition 2. A passive system

$$
G \equiv u_{t}+F\left(t, x, u, u_{1}, \ldots, u_{k}\right)=0, \quad H\left(t, x, u, u_{1}, \ldots, u_{m}\right)=0
$$

is called intermediate for equation

$$
u_{t x}+f\left(x, u, u_{1}, \ldots, u_{n}\right)=0,
$$

if

$$
D_{x}(G)+r H=u_{t x}+f
$$

where $r$ is a function of $t, x, u, u_{1}, \ldots, u_{p}(p<k)$.

We have given above two examples of intermediate systems. Now we will present one of the ways of constructing such systems.

Proposition 1. Let the operator

$$
Y=F\left(x, u, u_{1}, \ldots, u_{m}\right) \frac{\partial}{\partial u}+\sum_{n=1}^{\infty} D_{x}^{n}(F) \frac{\partial}{\partial u_{n}},
$$

where $\frac{\partial F}{\partial u_{m}} \neq 0$ is the higher symmetry of the equation (18). Then the system

$$
u_{t}+F=0, \quad D_{x}(F)-f=0
$$

is intermediate for the equation (18). 
Proof. The equality obviously holds

$$
u_{t x}+f=D_{x}\left(u_{t}+F\right)-\left(D_{x}(F)-f\right) .
$$

The equation (18) is invariant under a translation of $t$. As is well known, the symmetries of the equation form a Lie algebra $[8,10]$. Hence, according to the condition of the Proposition 1, the operator

$$
X=\left(u_{t}+F\right) \frac{\partial}{\partial u}+\sum_{n=1}^{\infty} D_{x}^{n}\left(u_{t}+F\right) \frac{\partial}{\partial u_{n}}
$$

is also a symmetry of the equation (18). Obviously, the operator $X$ is a symmetry of the equation $D_{x}\left(u_{t}+F\right)=0$ and this equation is invariant under the traslation. Hence, it admits an operator $Y$. As shown in [5], this means that the system (20) is passive.

As an example, consider the sine-Gordon equation (17). The operators of higher symmetries (19) for this equation form an infinite-dimensional Lie algebra $\mathcal{L}[10,11]$. In this case, the function $F$ is represented as a linear combination (with real coefficients) of the functions $F_{1}=u_{1}$, $F_{i+1}=L^{i}\left(F_{1}\right)$, where $L=D_{x}^{2}+u_{1}^{2}-u_{1} D^{-1} u_{2}$ is the so-called recurrence operator. In particular, the functions $F_{2}$ and $F_{3}$ are given by formulas

$$
F_{2}=u_{3}+\frac{1}{2} u_{1}^{3}, \quad F_{3}=u_{5}+\frac{5}{2}\left(u_{1}^{2} u_{3}+u_{1} u_{2}^{2}\right)+\frac{3}{8} u_{1}^{5} .
$$

As repeatedly noted, the Lie algebra $\mathcal{L}$ is also the algebra of symmetries of the mKdV equation

$$
u_{t}=u_{3}+\frac{1}{2} u_{1}^{3}
$$

Proposition 1 clarifies the connection between the solutions of higher order mKdV equations and the solutions of the sine-Gordon equation. The higher order $\mathrm{mKdV}$ equations are the evolution equations

$$
u_{t}+F=0,
$$

where the function $F$ is a linear combination of the functions $F_{1}=u_{1}, \ldots, F_{i+1}=L^{i}\left(F_{1}\right)$. According to Proposition 1, the system

$$
u_{t}+F=0, \quad D_{x}(F)+\sin (u)=0
$$

is passive and each of its solutions satisfies the sine-Gordon equation. In particular, the 1-soliton solution of the sine-Gordon equation

$$
u=4 \operatorname{arctg}(\exp (m x+t / m+n)), \quad m, n \in \mathbb{R}
$$

satisfies the system (22), where $F=c_{0} F_{3}+c_{1} F_{2}+c_{2} F_{1}$, and the constants $c_{0}, c_{1}, c_{2}, m$ are related by the formula $c_{0} m^{6}+c_{1} m^{4}+c_{2} m^{2}+1=0$.

In addition to the 1 -soliton solution, the intermediate system (22) has other solutions. As shown in the monograph [7], the standard numerical Runge-Kutta methods can be used to solve systems of the type (22). If we introduce new functions $u 1=u_{1}, \ldots, u 4=u_{4}$, then the system (22) with $F=c_{0} F_{3}+c_{1} F_{2}+c_{2} F_{1}$ is rewritten as two systems of ordinary differential equations of the first order in $t$ and $x$ respectively. Given five initial conditions, we solve the system of ordinary differential equations in $t$, and then use the obtained data to solve the system in terms of $x$. In this manner, we obtain a solution of the system (22) satisfying the initial data $u(0,0)=3.14$, $u_{1}(0,0)=2, u_{2}(0,0)=0, u_{3}(0,0)=-2, u_{4}(0,0)=0, u_{5}(0,0)=10$ and $c_{0}=-1, c_{1}=c_{2}=0$. The graph of the solution is shown in Fig. 1. 


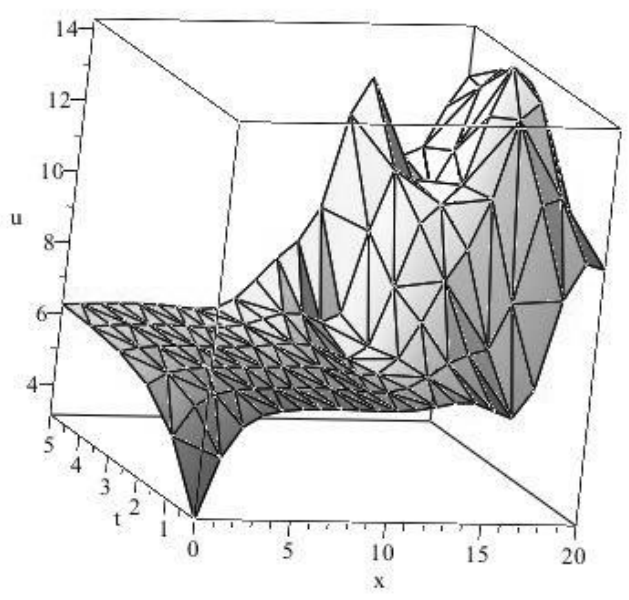

Fig. 1

\section{Intermediate systems of nonlinear wave equations}

In this section we construct examples of intermediate systems for the nonlinear wave equation

$$
u_{t x}+f(u)=0
$$

without using the symmetries. In the general case, the problem of finding intermediate systems is very difficult. It requires the solution of nonlinear partial differential equations with several independent variables.

We will look for intermediate systems in the form of polynomials with respect to $u_{i}(i>0)$ with coefficients depending on $u$. By analogy with (20), we assume that the system has the form

$$
\begin{gathered}
u_{t}=a_{1} u_{2}+a_{2} u_{1}^{2}, \\
h \equiv D_{x}\left(a_{1} u_{2}+a_{2} u_{1}^{2}\right)+f=0,
\end{gathered}
$$

where $a_{1}, a_{2}$ are only functions of $u$. The first equations in the system is invariant under a scale transformation of independent variables.

Since the system must be passive, it is proved in [7] that the function $h$ must satisfy the so-called linear defining equation of the form

$$
D_{t} h-r_{0} D_{x}^{2} h-r D_{x} h-r_{1} h=0,
$$

where $r_{0}, r, r_{1}$ are some functions of $u, u_{1}, \ldots, u_{n}$ and the relation (26) must be fulfilled because of the equation (24), i.e. the derivatives $u_{t}, u_{t x}, \ldots$ are expressed in terms of $a_{1} u_{2}+a_{2} u_{1}^{2}$, $D_{x}\left(a_{1} u_{2}+a_{2} u_{1}^{2}\right)$, and so on. The left hand side of the equation (26) is a polynomial with respect to $u_{1}, \ldots u_{5}$, and all its coefficients must therefore be zeros. Collecting similar terms for $u_{5}$ yields the relation $a_{1}^{2}-r_{0} a_{1}=0$. Hence, the formula holds

$$
r_{0}=a_{1}
$$

Next, we collect the terms with $u_{1} u_{4}$ and find the function $r$

$$
r=\left(a_{1}^{\prime}+2 a_{2}\right) u_{1} .
$$


If we collect similar terms containing $u_{3}$, then it is easy to express the function $r_{1}$

$$
r_{1}=2 u_{2}\left(a_{1}^{\prime}+a_{2}\right)+u_{1}^{2}\left(a_{2} a_{1}^{\prime} / a_{1}+3 a_{2}^{\prime}\right) .
$$

Collecting of similar terms with $u_{2}$ gives the relation

$$
a_{1}\left(a_{1}^{\prime}+a_{2}\right) f=0
$$

Since the case $a_{1} f=0$ is not of interest to us, the equality

$$
a_{2}=-a_{1}^{\prime}
$$

must hold. Collecting terms with $u_{1}^{2} u_{2}$ and taking into account the previous equality, we obtain the equation

$$
a_{1} a_{1}^{\prime \prime}=\left(a_{1}^{\prime}\right)^{2}
$$

which has a solution

$$
a_{1}=c \exp (k u)
$$

where $c, k$ are arbitrary constants. This yields

$$
a_{2}=-c k \exp (k u)
$$

Substituting the found functions into the defining equation (26) and collecting terms with $u_{1}^{2}$, we obtain the equation

$$
f^{\prime \prime}-4 k^{2} f=0
$$

From the equation we find

$$
f=c_{1} \exp (2 k u)+c_{2} \exp (-2 k u), \quad c_{1}, c_{2} \in \mathbb{R} .
$$

Thus, the intermediate system (24), (25) has the form

$$
u_{t}=c e^{k u}\left(u_{2}-k u_{1}^{2}\right), \quad h \equiv D_{x}\left(c e^{k u}\left(u_{2}-k u_{1}^{2}\right)\right)+c_{1} e^{2 k u}+c_{2} e^{-2 k u}=0,
$$

and the corresponding equation (23) is

$$
u_{t x}=c_{1} e^{2 k u}+c_{2} e^{-2 k u}
$$

If $c=k=1$, then the linear defining equation (26) is

$$
D_{t} h-e^{u}\left(D_{x}^{2} h-u_{1} D_{x} h-4 u_{1}^{2} h\right)=0 .
$$

Now consider the third-order evolution equation

$$
u_{t}=F \equiv a_{1} u_{3}+a_{2} u_{1} u_{2}+a_{3} u_{1}^{3},
$$

with $a_{1}, a_{2}, a_{3} \in \mathbb{R}$. The additional equation is given by the formula

$$
h \equiv D_{x} F+f=0 .
$$

The system (27), (28) must be passive and, consequently, satisfy a linear defining equation of the form

$$
D_{t} h-s D_{x}^{3} h-r_{0} D_{x}^{2} h-r D_{x} h-r_{1} h=0,
$$

where $s, r_{0}, r, r_{1}$ are functions of $u, u_{1}, u_{2}, u_{3}$ to be determined together with $h$. 
The left-hand side $(29)$ is a polynomial in $u_{1}, \ldots, u_{7}$. All the coefficients of this polynomial must be zero. It is easy to calculate that the coefficient of $u_{7}$ is equal to $a_{1}^{2}-s a_{1}$. It follows that $s=a_{1}$. Collecting terms with $u_{6}, u_{5}, u_{4}$, we find the functions $r_{0}, r, r_{1}$ :

$$
\begin{gathered}
r_{0}=\left(a_{1}^{\prime}+a_{2}\right) u_{1}, \quad r=\left(a_{2}^{\prime}+3 a_{3}\right) u_{1}^{2}+2 a_{2} u_{2}, \\
r_{1}=\left[u_{3} a_{1}\left(2 a_{1}^{\prime}+a_{2}\right)+u_{1} u_{2}\left(a_{1}^{\prime} a_{2}+2 a_{1} a_{2}^{\prime}+6 a_{1} a_{3}\right)+u_{1}^{3}\left(a_{1}^{\prime} a_{3}+4 a_{1} a_{3}^{\prime}\right)\right] / a_{1} .
\end{gathered}
$$

The remaining terms on the left hand side (29) form a polynomial in three variables $u_{1}, u_{2}, u_{3}$. Equating the coefficients of this polynomial to zero, we obtain an overdetermined system of ordinary differential equations for functions $a_{1}, a_{2}, a_{3}, f$. This system is easy to solve. Omitting the computational details, we see that there are only three types of solutions. We present the final form of the equations (27) and the corresponding functions $f$ :

$$
\begin{array}{lll}
\text { (1) } u_{t}=e^{u}\left(u_{3}-2 u_{1} u_{2}\right), & f=c_{1} e^{2 u}+c_{2} e^{-u}, \\
\text { (2) } u_{t}=u_{3}-u_{1}^{3} / 2, & f=c_{1} e^{u}+c_{2} e^{-u,} \\
\text { (3) } u_{t}=u_{3}+u_{1}^{3} / 2, & f=c_{1} \sin (u)+c_{2} \cos (u),
\end{array}
$$

where $c_{1}, c_{2}$ are arbitrary constants. The second and third evolution equations are well known, but the first one is probably new.

\section{Diffusion equations with constraints}

In this section, we will continue constructing passive systems using the defining equations. Let us consider a nonlinear diffusion equation

$$
v_{t}=\left(v^{k} v_{x}\right)_{x}, \quad k \in \mathbb{R} .
$$

We replace $u$ by $v^{k}$ and obtain an equation with the bilinear operator [13]

$$
u_{t}=u u_{x x}+b u_{x}^{2}, \quad b=1 / k .
$$

As in the previous sections, we supplement the equation (30) by an ordinary differential equation

$$
h=0,
$$

the right-hand side of which satisfies the linear defining equation

$$
D_{t} h-r_{0} D_{x}^{2} h-r D_{x} h-r_{1} h=0,
$$

where $r_{0}, r, r_{1}$ are functions of $u, u_{1}, u_{2}, \ldots$. We assume that $h$ has the form

$$
h=u_{3}+a_{1} u_{1} u_{2}+a_{2} u_{1}^{3}+a_{3} u_{1}+a_{4} .
$$

Here $a_{1}, a_{2}, a_{3}, a_{4}$ are only functions of $u$. The left-hand side of the equation (31) is a polynomial in $u_{1}, \ldots, u_{5}$. Equating the coefficients of this polynomial containing $u_{5}, u_{4}, u_{3}$ to zero, we have

$$
r_{0}=u, \quad r=(2 b+3) u_{1}, \quad r_{1}=\left(2 a_{1}^{\prime} u-a_{1}\right) u_{1}^{2}+\left(6 b+4-2 a_{1} u\right) u_{2} .
$$

The remaining terms in the polynomial lead to an overdetermined system of ordinary differential equations containing unknown functions $a_{1}, \ldots, a_{4}$. Omitting the long but easy calculations, we give the final result. 
Proposition 2. For any $b \in \mathbb{R}$ the linear defining equation has two solutions

$$
h_{1}=u_{3}, \quad h_{2}=u_{3}+2 b u_{1} u_{2} / u-b u_{1}^{3} / u^{2} .
$$

Moreover, there are additional solutions of (31) for the following values of $b$ :

$$
\begin{array}{ll}
\text { (a) } b=-1, & h=u_{3}+c u_{1}, \quad h=u_{3}+\frac{2 u u_{1} u_{2}-u_{1}^{3}-c_{1} u_{1}}{2 c_{2}-u^{2}}, \\
\text { (b) } b=-2, & h=u_{3}-\frac{4 u_{1} u_{2}}{u}+2 \frac{u_{1}^{3}}{u^{2}}+c_{1} u^{2}+2 \frac{c_{2}}{u^{2}}, \\
\text { (c) } b=-2 / 3, & h=u_{3}+c_{1} u^{-4 / 3}+c_{2} \\
\text { (d) } b=-1 / 2, & h=u_{3}+\frac{u_{1}\left(u_{2}+2 c_{1}\right)}{u\left(c_{2} u^{1 / 2}+2\right)}
\end{array}
$$

where $c, c_{1}, c_{2}$ are arbitrary constants.

Remark. Some solutions of the equation (30) are given in $[5,13,14]$. In particular, for $b=-1 / 2$ this equation can be linearized.

As an example, we show how to find exact solutions of the system

$$
u_{t}=u u_{2}-u_{1}^{2}, \quad u_{3}+\frac{2 u u_{1} u_{2}-u_{1}^{3}-c_{1} u_{1}}{2 c_{2}-u^{2}}=0 .
$$

We rewrite the second equation in the form

$$
u_{3}\left(2 c_{2}-u^{2}\right) / u_{1}+2 u u_{2}-u_{1}^{2}=c_{1}
$$

and differentiate with respect to $x$. This leads to the fourth order differential equation

$$
u_{4} u_{1}-u_{2} u_{3}=0
$$

without any constants! Dividing the equation by $u_{1} u_{3}$ and integrating, we obtain

$$
u_{3}+c u_{1}=0 .
$$

If $c=-k^{2}$, then the general solution of the last equation is

$$
u=s_{1} e^{k x}+s_{2} e^{-k x}+s_{3},
$$

where $k, s_{1}, s_{2}, s_{3}$ are functions of $t$. We substitute this solution in the second equation of the system (33) and find

$$
s_{1}=\frac{c_{1}-2 c_{2} k^{2}+k^{2} s_{3}^{2}}{4 k^{2} s_{2}}
$$

Next, we substitute the function $u$ into the first equation of the system (33). As a result, we have the system of equations

$$
k^{\prime}=0, \quad s_{2}^{\prime}=k^{2} s_{2} s_{3}, \quad s_{2} s_{2}^{\prime \prime}-2\left(s_{2}^{\prime}\right)^{2}=\left(c_{1} k^{2}-2 c_{2} k^{4}\right) s_{2}^{2} .
$$

If the constant $m=-c_{1} k^{2}+2 c_{2} k^{4}$ is positive, then

$$
s_{2}=\frac{1}{d_{1} e^{t \sqrt{m}}+d_{2} e^{-t \sqrt{m}}}, \quad d_{1}, d_{2} \in \mathbb{R} ;
$$

otherwise, we obtain

$$
s_{2}=\frac{1}{d_{1} \sin (t \sqrt{-m})+d_{2} \cos (t \sqrt{-m})} .
$$


The functions $s_{1}, s_{3}$ are expressed from the above formulas.

Another solution of the defining equation (31) is

$$
h \equiv u_{3}-\frac{u_{2}^{2}}{u_{1}}+\frac{(b+1) u_{1} u_{2}}{u}=0 .
$$

Moreover, the system (30), (34) is passive for any $b \in \mathbb{R}$. If we divide $h$ by $u_{2}$, then it is easy to find the first integral and then reduce it to the first-order equation

$$
u_{1}=q_{1} u^{-b}+q_{2},
$$

where $q_{1}, q_{2}$ are functions of $t$. For some values of $b$, we can construct solutions of the last equation in elementary functions. These solutions are related to the invariance of the equation (30) under the translations in $t$ and $x$.

We give an additional statement which is verified by direct calculations.

Proposition 3. The equations (30) and $h=0$ form a passive system with the following values of the constant $b$ and the function $h$ :

$$
\begin{aligned}
& \forall b \in \mathbb{R} \quad h=u_{4}-\frac{3 u_{2} u_{3}}{u_{1}}+\frac{(b+2) u_{1} u_{3}}{u}+\frac{2 u_{2}^{3}}{u_{1}^{2}}-\frac{u_{2}^{2}}{u} ; \\
& b=-1, \quad h=u_{4}-\frac{u_{2} u_{3}}{u_{1}} \\
& b=-2, \quad h=u_{4}-\frac{6 u_{1} u_{3}}{u}-\frac{4 u_{2}^{2}}{u}+\frac{18 u_{1}^{2} u_{2}}{u^{2}}-\frac{8 u_{1}^{4}}{u^{3}} ; \\
& b=-2, \quad h=u_{4}-\frac{2 u_{1} u_{3}}{u}-\frac{4 u_{2}^{2}}{u}+\frac{2 u_{1}^{2} u_{2}}{u^{2}} ; \\
& b=-2 / 3, \quad h=u_{4}+\frac{4 u_{1} u_{3}}{3 u} ; \\
& b=-1 / 2, \quad h=u_{4}+\frac{u_{1} u_{3}}{u}+\frac{u_{2}^{2}}{2 u}-\frac{u_{1}^{2} u_{2}}{4 u^{2}} ; \\
& b=-1 / 2, \quad h=u_{4}+\frac{3 u_{1} u_{3}}{2 u}+\frac{u_{2}^{2}}{2 u} ; \\
& b=1 / 3, \quad h=u_{5}+\frac{1}{3 u u_{1}}\left(3 u u_{2} u_{4}+7 u_{1}^{2} u_{4}\right) ; \\
& b=-1, \quad h=u_{5}+\frac{1}{u^{3} u_{1}}\left(-u^{3} u_{2} u_{4}-3 u_{4} u_{1}^{2} u^{2}-8 u_{3} u_{2} u_{1} u^{2}+\right. \\
& \left.+6 u_{3} u_{1}^{3} u+4 u_{2}^{3} u^{2}+10 u_{2}^{2} u_{1}^{2} u-6 u_{2} u_{1}^{4}\right) \\
& b=-1 / 2, \quad h=u_{5}+\frac{1}{2 u}\left(u_{1} u_{4}-15 u_{2} u_{3}\right) ; \\
& b=1 / 2, \quad h=u_{5}+\frac{1}{4 u^{2}}\left(12 u u_{1} u_{4}+10 u_{3} u_{2} u+3 u_{3} u_{1}^{2}+u_{2}^{2} u_{1}\right) ; \\
& b=1 / 4, \quad h=u_{5} \text {; } \\
& b=1 / 2, \quad h=u_{5}+\frac{1}{2 u}\left(5 u_{1} u_{4}+5 u_{2} u_{3}\right) .
\end{aligned}
$$

The work was supported by the Russian Foundation for Basic Research (grant 17-01-00332-a).

\section{References}

[1] A.F.Sidorov, V.P.Shapeev, N.N.Yanenko, The method of differential constraints and its applications in gas dynamics, Novosibirsk, Nauka, 1984 (in Russian). 
[2] V.I.Smimov, Course of high mathematics, NY, PERGAMON PRESS, 1964.

[3] M.E.Goursat, Recherches sur quelques équations aux dérivées partielles du second order, Annales de la Faculté de Toulouse, 2 série, t. 1, 1899.

[4] A.V.Zhiber, R.D.Murtazina, I.T.Habibullin, A.B.Shabat, Characteristic Lie rings and integrable models in mathematical physics, Ufimsk. Mat. Zh.,4(2012), no. 3, 17-85 (in Russian).

[5] O.V.Kaptsov, Integration Methods for Partial Differential Equations, Fizmatlit, Moscow, 2009 (in Russian).

[6] O.V.Kaptsov, On the Goursat classification problem, Programming and Computer Software, 32(2012), no. 2, 68-71.

[7] V.K.Andreev, O.V.Kaptsov, V.V.Pukhnachev, A.A.Rodionov, Applications of GroupTheoretical Methods in Hydrodynamics, Springer, Netherlands, 2010.

[8] Symmetries and Conservation Laws for Differential Equations of Mathematical Physics, Editors: I. S. Krasilshchik, A. M. Vinogradov, AMS, 1999.

[9] P.Olver, Applications of Lie Groups to Differential Equations, NY, Springer-Verlag, 1993.

[10] N.H.Ibragimov, Transformation Groups Applied to Mathematical Physics, Kluwer AP, Dordrecht, 1985.

[11] A.V.Zhiber, A.B.Shabat, Klein-Gordon equations with a nontrivial group, Sov. Phys. Dokl., 24(1979), no. 8, 607-609.

[12] O.V.Kaptsov, Algebraic and geometric structures of analytic partial differential equations, TMP, 189(2016), no. 2, 1592-1608.

[13] V.A.Galaktionov, S.R.Svirshchevskii, Exact Solutions and Invariant Subspaces of Nonlinear Partial Differential Equations in Mechanics and Physics, Chapman \& Hall, London, 2007.

[14] A.D.Polyanin, V.F.Zaitsev, Handbook of Nonlinear Partial Differential Equations, CRC Press, 2004.

\section{Промежуточные системы и дифференциальные связи высших порядков}

Олег В. Капцов

Институт вычислительного моделирования СО РАН Академгородок, 50/44, Красноярск, 660036

Россия

$\overline{B \text { работе предложен метод построения решений нелинейных уравнений в частных производных } c}$ двумя независимыми переменными. Метод основан на поиске так называемых промежуточных систем, каждое решение которых удовлетворяет исходному уравнению. Основное внимание уделяется нелинейному волновому уравнению второго порядка. Приведены примеры промежуточных систем и соответствующих решений.

Ключевые слова: дифференциальные связи, определяющие уравнения, инвариантные многообразия. 